\title{
La inhibición de Rho quinasa post infarto mejora el remodelado y la función ventricular: mecanismos involucrados a nivel preclínico
}

\author{
Claudia Mera, Iván Godoy, Renato Ramírez, Jackeline Moya, María Paz Ocaranza, Jorge E Jalil \\ Pontificia Universidad Católica de Chile, Escuela de Medicina \\ Departamento de Enfermedades Cardiovasculares, \\ Laboratorio de Cardiología Molecular \\ Financiamiento a través de Grant para Investigador Joven, Sociedad Chilena de Cardiología; Grants Fondecyt \\ 1085208 y 1121060 y Fondap 15130011.
}

Objetivo: Determinar algunos mecanismos moleculares por los cuales la activación de ROCK cardíaca post infarto del miocardio (IAM) participa en el remodelado y en deterioro de la función sistólica.

Métodos: Determinación simultánea de niveles de proteínas blanco de ROCK cardíaca, de función sistólica in vivo del ventrículo izquierdo (VI) y de fibrosis e hipertrofia cardíaca en ratas con IAM en condiciones de inhibición de ROCK con fasudil.

Resultados: Siete días post IAM la masa ventricular relativa aumentó significativamente en un $30 \%$ en el grupo MI y se redujo con fasudil. La disfunción sistólica VI mejoró significativamente con fasudil mientras que la activación de ROCK cardíaca se redujo a niveles del grupo control. El inhibidor de ROCK también redujo significativamente los niveles cardíacos elevados de las isoformas ROCK1 y ROCK2, de MHC- $\beta$ y del colágeno miocárdico. En el grupo con IAM aumentaron significativamente los niveles de fosforilación de ERK 42 y ERK 44 (en 2 veces y en 63\%, respectivamente), mientras que en el grupo IAM tratado con fasudil estos niveles fueron similares a los del grupo control. El IAM aumentó significativamente los niveles fosforilados del factor de transcripción GATA4 , que se normalizaron con el inhibidor de ROCK.

Conclusiones: La disfunción sistólica post IAM se asoció fuertemente con la activación del ROCK cardíaca y con la fosforilación de proteínas río abajo de ROCK que promueven remodelado cardíaco como $\beta$-MHC y la vía ERK / GATA-4.

\section{Correspondencia:}

Dr. Jorge E Jalil

Pontificia Universidad Católica de Chile, Escuela de Medicina

Departamento de Enfermedades Cardiovasculares,

Lira 85 Piso 2, Santiago.

jjaliı@med.puc.cl 


\section{Rho-kinase inhibition post myocardial infarction improves remodeling and systolic function: a preclinical study of intervening factors in a pre-clinical study}

Objective: to determine some molecular mechanisms by which cardiac ROCK activation after myocardial infarction (MI) intervene in cardiac systolic function decline and remodeling.

Methods: simultaneous measurement of different cardiac ROCK target proteins levels, in vivo left ventricular (LV) systolic function, myocardial fibrosis, and hypertrophy in rats with MI under ROCK inhibition with fasudil were performed.

Results: seven days after MI the relative ventricular mass increased significantly by $30 \%$ in the MI group and was reduced with fasudil. LV systolic dysfunction improved significantly with fasudil whereas at the same time cardiac ROCK activation was reduced to sham levels. The ROCK inhibitor also reduced increased cardiac levels of both ROCK1 and ROCK2 isofor$\mathrm{ms}, \beta$-MHC levels and myocardial collagen volume fraction decline. MI significantly increased phosphorylation levels of ERK 42 and ERK 44 by 2 -fold and $63 \%$ respectively whereas in the fasudil-treated MI group these levels were similar to those in the sham group. MI significantly increased phosphorylated levels of the transcription factor GATA-4 which were normalyzed by the ROCK inhibitor.

Conclusion: LV systolic dysfunction after MI was strongly associated to cardiac ROCK activation and subsequent phosphorylation of ROCK target proteins that promote ventricular remodeling, such as $\beta$-MHC and the ERK/GATA-4 pathway. ROCK inhibition with fasudil significantly improved systolic function, diminished myocardial fibrosis, and normalized $\beta$-MHC and ERK/GATA-4 phosphorylation levels.

Keywords: Contractility, ERM, Fasudil, Heart failure, Rho kinase, Remodeling. 


\section{Introducción:}

A nivel global el infarto del miocardio (IAM) es una de las causas principales de insuficiencia cardíaca (IC) y de mortalidad en la población. Las modificaciones cardíacas post IAM y el remodelado son mecanismos compensatorios que mantienen inicialmente la integridad del tejido y preservan la función ventricular. Cuando esta respuesta adaptativa temprana es progresiva y mantenida en el tiempo, el remodelado cardíaco resulta adverso y necesariamente lleva a disfunción ventricular y a $\mathrm{IC}^{1-3}$. En la fase aguda post IAM, la liberación de mediadores inflamatorios activa vías de señalización intracelular en el miocardio. Estas vías incluyen respuestas neurohormonales que restablecen la presión arterial y el flujo sanguíneo, alteran la expresión de proteínas miocárdicas o modifican el nivel de fosforilación de proteínas y, de esta manera, su función ${ }^{4}$.

Una de las vías de señalización asociada al remodelado patológico y a la disfunción contráctil post IAM es la vía RhoA y su principal proteína blanco Rho quinasa (ROCK). RhoA se activa mediante agonistas de receptores acoplados a proteína $\mathrm{G}$ de la membrana celular, como Ang II y noradrenalina entre otros ${ }^{5}$. RhoA activada se transloca a la membrana celular, donde fosforila y por lo tanto activa a ROCK. ROCK activada media importantes funciones celulares como son la contracción muscular, organización del citoesqueleto, adhesión y motilidad, proliferación, diferenciación, apoptosis, supervivencia y expresión de genes implicados en remodelado ${ }^{6-12}$. ROCK también interviene en la regulación positiva de varias moléculas proinflamatorias, trombogénicas y fibrogénicas, y regula negativamente el óxido nítrico sintasa endotelial. Además, la activación de ROCK promueve el estrés oxidativo vascular ${ }^{13}$.

Estudios preclínicos dan cuenta del rol de la activación de ROCK en procesos clave asociados con remodelado post IAM $^{14-22}$. En ratones que sobre expresan Gaq, la deleción del gen $\mathrm{ROCK}^{1}$ previene la dilatación ventricular y la disfunción sistólica ${ }^{23}$. En cardiomiocitos cultivados, la activación de ROCK up regula a Bax, lo que induce muerte mitocondrial y apoptosis de los cardiomiocitos 24 . En ratones transgénicos que sobre expresan la subunidad 2 de la fosfatasa de miosina (blanco de ROCK), una mayor actividad de esta fosfatasa produce disfunción y dilatación $\mathrm{VI}^{25}$.

Aún así, no está del todo claro si la disfunción sistólica post IAM se relaciona con la fosforilación de proteínas que son blanco de ROCK y que promueven remodelado ventricular y cual es el rol de la inhibición de ROCK es en este contexto. El objetivo del presente estudio fue evaluar algunos mecanismos moleculares a través de los cuales la activación cardíaca de ROCK post IAM participa en el remodelado y en el deterioro de la función ventricular, determinando simultáneamente niveles de proteínas río abajo de ROCK, función sistólica VI in vivo además de niveles de fibrosis e hipertrofia miocárdica en ratas con inhibición farmacológica de ROCK. El trabajo presenta los hallazgos más significativos previamente publicados por nosotros 27 .

\section{Métodos:}

El trabajo se realizó de acuerdo con la "Guía para el cuidado y uso de animales de laboratorio" (NIH No. 85-23, revisado en 1996) y fue aprobado previamente por el Comité de Ética y Bienestar Animal de la Facultad de Medicina de la Pontificia Universidad Católica de Chile. Modelo experimental de infarto del miocardio. Se obtuvieron ratas macho adultas Sprague-Dawley (peso $200 \pm$ $10 \mathrm{~g}$, de 9 semanas) que se mantuvieron bajo ciclos de luz /oscuridad de 12 horas a temperatura y humedad reguladas con acceso libre a comida y agua. El IAM se indujo por ligadura de la arteria descendente anterior izquierda (por toracotomía izquierda) bajo anestesia intraperitoneal (ip) con ketamina (50 mg / kg) y xilazina (10 mg / kg) y ventilación mecánica. En el grupo control, la cirugía se realizó de la misma manera, pero sin ligadura de la arteria coronaria. Las ratas infartadas se dividieron aleatoriamente en 2 grupos: uno con y otro sin tratamiento oral con fasudil, inhibidor farmacológico de ROCK (100 $\mathrm{mg} / \mathrm{kg} /$ día por gavage, 1 semana) iniciado un día después de la cirugía.

Función ventricular izquierda (ecocardiografía bidimensional). Una semana después de la ligadura coronaria, un ecocardiografista experimentado realizó ciegamente un ecocardiograma bajo sedación con ketamina y xilazina (equipo Sonos 5500 Philips y un transductor de $12 \mathrm{MHz}$. Se obtuvo una visión paraesternal del eje corto del ventrículo izquierdo (VI) y se determinaron: diámetro telesistólico (DS), diámetro telediastólico (DD), grosor de la pared anterior y posterior y fracción de acortamiento del VI. La fracción de eyección (FE) se cuantificó con la metodología descrita por de Simone et $\mathrm{al}^{26}$.

Morfometría miocárdica. En el VI se determinó hipertrofia cardiomiocitaria y fibrosis miocárdica. Una semana post IAM, las ratas fueron eutanasiadas. Se extrajo cuidadosamente cada corazón, se lavó en solución salina, se 
pesó, y fijó en formalina al 4\% para inclusión en parafina. Los corazones fijados se cortaron transversalmente (5 $\mu \mathrm{m})$ y se tiñeron con hematoxilina-eosina (HE).

Se analizaron las secciones transversales de los cardiomiocitos en la pared VI no infartada, bajo microscopio óptico (Nikon Eclipse E400) a 400X. Se midieron cien células de cada roedor en todo el miocardio y endocardio del VI. Se delimitó el perímetro de cada celda seleccionada para calcular el área de sección transversal usando el software NIS-Element BR 3.0.

Las imágenes para determinar la fracción de volumen de colágeno intersticial (CVF) fueron adquiridas en un microscopio óptico (Nikon Eclipse E400). De cada VI se examinaron 20 imágenes correspondientes al área infartada, al área cercana al infarto y al área lejos del infarto (zona en la sección transversal ubicada exactamente a $180^{\circ}$ del centro de la cicatriz) con aumento de $200 \mathrm{X}$. El colágeno se cuantificó mediante morfometría asistida por computadora utilizando el software MATLAB6.1.

Cuantificación de proteínas (Western Blot). Para el análisis de Western blot, se congelaron muestras de todo el VI en nitrógeno líquido y se mantuvieron a $-80^{\circ} \mathrm{C}$ hasta su procesamiento. Las muestras de VI se homogeneizaron y se lisaron con tampón de lisis con bajas concentraciones de detergente suplementado con inhibidores de proteasa e inhibidores de fosfatasas en hielo ${ }^{27}$. Se cargaron cantidades iguales de proteína, se separaron en un gel SDS-PAGE al 10\% y se transfirieron a una membrana de nitrocelulosa. Después de bloquear con leche descremada (para proteínas no fosforiladas) o BSA 5\% (para proteínas fosforiladas) durante 1 hora a temperatura ambiente, las transferencias se incubaron durante la noche a $4^{\circ} \mathrm{C}$ con los siguientes anticuerpos: anti subunidad blanco de la fosfatasa de la miosina-1 (MYPT-1, ratón monoclonal, 1/1000 BD Biosciences); anti p-MYPT-1 (Phospho-MYPT1-Thr 853 Conejo policlonal, 1/700, Cyclex Co), anti Ezrina-radixina-moesina (ERM, Total-ERM, conejo policlonal, 1/700, Cell Signaling); anti p-ERM (Phospho-ERM, Ezrin Thr567, Radixin Thr564, Moesin Thr558, Conejo policlonal, 1/700, Cell Signaling); anti $\beta$-MHC (cadena pesada de miosina, monoclonal de ratón, 1/1000, Novocastra TM); anti $\alpha$-SKA (ratón monoclonal, 1/2500, US Biological Life Sciences); anti p-Smad3 (Phospho-Smad S423 / 425, monoclonal de conejo, 1/1000, Cell Signaling); anti Smad3 (Total-Smad, conejo monoclonal, 1/1000, Cell Signaling); anti ROCK1 (ratón monoclonal, 1/1000, BD BioScience); anti ROCK-2 (monoclonal de ratón, 1/1000, BD BioScience); anti ERK1/2 (Total-ERK, conejo policlonal, 1/1000, Cell Signaling); anti p-ERK1 / 2 (Phospho-ERK 44/42 Thr202 / Tyr204, 1/1000, Cell Signaling); anti GATA-

\begin{tabular}{|c|c|c|c|}
\hline & Control (Sham) & IAM & IAM+ Fasudil \\
\hline N & 5 & 7 & 7 \\
\hline \multicolumn{4}{|l|}{ Parámetros Ecocardiográficos } \\
\hline Area infartada (\%) & $\ldots$ & 29,4 & 26,8 \\
\hline Grosor de pared anterior VI ( $\mathrm{mm})$ & $1,46 \pm 0,02$ & $0,87 \pm 0,02 \#$ & $0,83 \pm 0,04 \#$ \\
\hline Dimensión sistólica VI (mm) & $4.9 \pm 0.2$ & $7.8 \pm 0.2 \#$ & $7.2 \pm 0.2 \# @$ \\
\hline Dimensión diastólica VI (mm) & $7.1 \pm 0.2$ & $8.7 \pm 0.2 \#$ & $8.5 \pm 0.3 \#$ \\
\hline Fracción de acortamiento VI (\%) & $28,7 \pm 1,7$ & $10,1 \pm 0,7 \#$ & $15,3 \pm 1,2$ \# @ \\
\hline Fracción de eyección VI (\%) & $51,3 \pm 3,0$ & $19,7 \pm 1,7 \#$ & $30,1 \pm 1,6$ \# @ \\
\hline \multicolumn{4}{|l|}{ Masa cardíaca } \\
\hline Peso corporal (g) & $243 \pm 11,6$ & $203 \pm 15,7$ & $202 \pm 12,4$ \\
\hline Peso cardíaco (g) & $0,88 \pm 0,02$ & $0,93 \pm 0,03 \#$ & $0,77 \pm 0,01 \#$ \\
\hline Masa cardíaca relativa $(\mathrm{mg} / \mathrm{g})$ & $0,36 \pm 0,01$ & $0,47 \pm 0,03 \#$ & $0,39 \pm 0,02 @$ \\
\hline
\end{tabular}


4 (Total-GATA 4, conejo policlonal, 1/1000, Thermo Scientific) y anti p-GATA-4 (Phospho-GATA 4, fosfo S105, 1/1000, conejo policlonal). A continuación, los blots se lavaron e incubaron con un anticuerpo secundario de cabra anti-IgG de conejo conjugado con HRP (1: 5000, Thermo Scientific) o uno de cabra anti IgG de ratón (1: 10.000, Santa Cruz) durante dos horas. La cantidad relativa de proteína se estimó mediante quimioluminiscencia usando el kit ECL plus (Perkin Elmer). Las imágenes digitales obtenidas se analizaron por densitometría utilizando el software Image J (NIH, EE.UU.). Como proteína de control de carga (GADPH) se usó un anticuerpo monoclonal de ratón anti GAPDH (1: 1000) (Santa Cruz Biotechnology Inc).

Análisis estadístico. Los datos se presentan como promedio \pm ES. Los promedios se compararon con análisis de varianza de un factor (ANOVA) seguido del test de Newman-Keuls. Un valor de $\mathrm{p}<0.05$ se consideró estadísticamente significativo.

\section{Resultados:}

Características de los grupos experimentales y función ventricular izquierda (Tabla 1). Una semana post IAM, el peso corporal disminuyó en el grupo IAM y en el grupo IAM+fasudil en comparación con el grupo control operado, sin ser significativo. La masa ventricular (peso de ambos ventrículos) aumentó significativamente en el grupo IAM en $6 \%$, y en las ratas con IAM tratadas con fasudil la masa ventricular disminuyó significativamente en $18 \%$ en comparación el grupo IAM (y $13 \%$ en comparación con el grupo control). La masa ventricular relativa (masa ventricular/peso corporal) aumentó significativamente en $30 \%$ en el grupo IAM y disminuyó significativamente con fasudil.

Dimensiones y función sistólica VI (Tabla 1). En el grupo IAM se observó una dilatación importante de la cavidad VI. El área de fin de sístole se incrementó significativamente 2,6 veces en el grupo IAM en comparación con el grupo control y disminuyó en $40 \%$ con fasudil. Al mismo tiempo, la fracción de acortamiento (FAC) y la fracción de eyección del VI (EF) se vieron gravemente comprometidas en el grupo IAM, lo que mejoró significativamente con la inhibición farmacológica de ROCK.

Activación de la vía RhoA/ROCK en miocardio. La activación de la ROCK cardíaca se evaluó mediante la determinación de los niveles de fosforilación de sus dos
Figura 1.
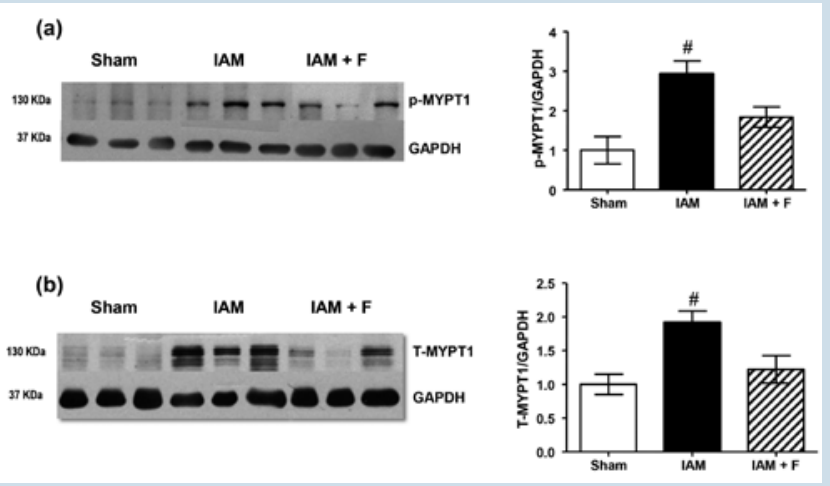

Activación de ROCK cardíaca post IAM evaluada por fosforilación de MYPT1. (a) Izquierda: Imágenes representativas de WB para p-MYPT1 de VI en ratas 1 semana post IAM en los 3 grupos experimentales (Sham, $n=5$; IAM, $n=7$ e IAM tratado con fasudil, $n=6$ ). Derecha: Análisis densitométrico en los 3 grupos experimentales. (b) Derecha: Imágenes representativas de WB para T-MYPT1 de VI en ratas 1 semana post IAM en los 3 grupos experimentales (Sham, IAM, e IAM tratado con fasudil). (c) Análisis densitométrico de la relación p-MYPT1/T-MYPT1. Las cuantificaciones se expresan en relación con el grupo Sham (en número de veces). Las determinaciones se normalizaron con los niveles de GAPDH. Los datos se expresan como promedio \pm ES. Símbolos: \# = $p<0.01$ vs Sham (Newman Keuls post ANOVA significativo).

Figura 2.
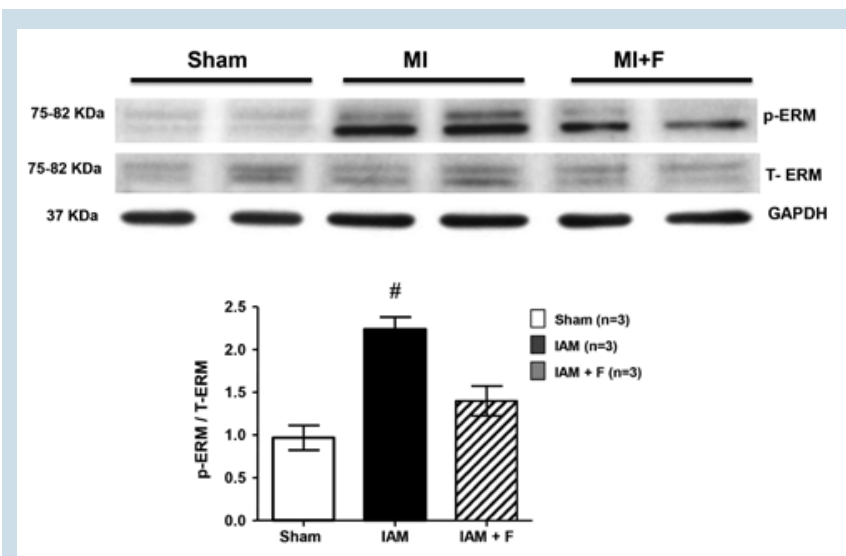

Activación de ROCK cardíaca post IAM evaluada por fosforilación de ERM. Western blots (fosforilado y total) y los análisis densitométricos respectivos de $\mathrm{VI}$ en ratas 1 semana post IAM en los 3 grupos experimentales (Sham, IAM, e IAM tratado con fasudil; $n=3 / g r u p o)$. La dependencia de la activación de ROCK se evaluó administrando fasudil. Cuantificaciones expresadas en relación con el grupo Sham (en número de veces). Las determinaciones están normalizadas con los niveles de GAPDH. Datos expresados como promedio \pm ES. Símbolos: $\#=p<0.01$ vs Sham (Newman Keuls post ANOVA significativo). 
principales sustratos directos, MYPT-1 y ERM en el miocardio del VI no infartado. La fosforilación de MYPT-1 (p-MYPT1) en el residuo 853 es específica para ROCK. En ratas con IAM, p-MPYT1 aumentó significativamente en $2.9 \pm 0.3$ veces en comparación con el grupo control, y se redujo significativamente con la administración de fasudil (Figura 1a). Los niveles de proteína total de MYPT1 (T-MYPT1) en ratas infartadas aumentaron 1,9 $\pm 0,2$ veces y también disminuyeron significativamente con fasudil (Figura 1b). Los niveles de p-MYPT1/T-MYTP1 en el grupo IAM aumentaron significativamente en $60 \%$ y se mantuvieron altos después de la administración de fasudil. Con el fin de precisar el nivel de activación de ROCK cardiaco, se determinaron también los niveles de fosforilación de un segundo blanco directo de ROCK, el complejo ERM, en el VI. En las ratas infartadas se observó un aumento significativo de los niveles de p-ERM/T-ERM cardíaco en 2,2 $\pm 0,14$ veces y se redujeron significativamente con fasudil (Figura 2).

Isoformas de ROCK (Figura 3). Los niveles proteicos de las isoformas ROCK1 y ROCK2 en miocardio post IAM aumentaron significativamente en $50 \%$ (p<0.01) y $40 \%$ $(\mathrm{p}<0.05)$, respectivamente post IAM. Fasudil redujo significativamente los niveles cardíacos de ambas isoformas.

Niveles cardíacos de $\beta$-MHC y $\alpha$-SKA y área de los cardiomiocitos (Figura 4). Los niveles de proteína de $\beta$-MHC y $\alpha$-SKA se usaron como marcadores tempranos de hipertrofia cardiomiocitaria. Los niveles de $\beta$-MHC

\section{Figura 3}

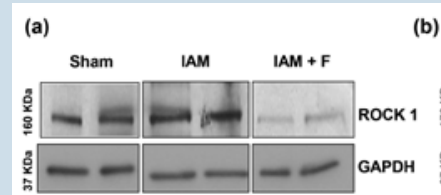

(b)

(a)
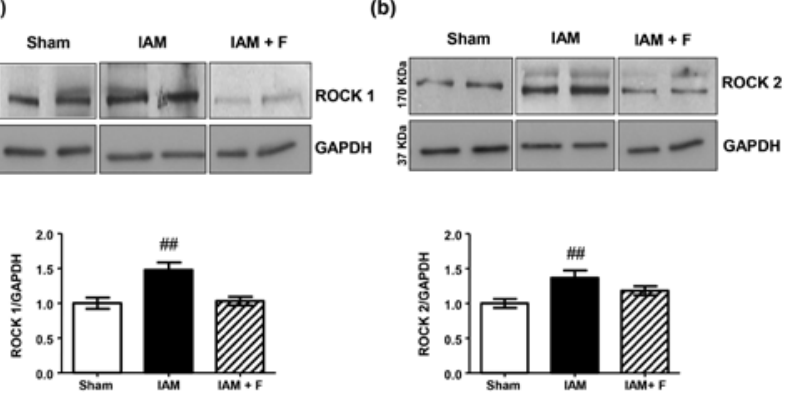

Fasudil disminuye la expresión proteica de ROCK1 y ROCK2 en el miocardio post IAM. Imágenes representativas de WB y análisis densitométrico de (a) ROCK 1 y (b) ROCK 2, de Vl en ratas en los 3 grupos experimentales (Sham, $n=8 ; \mid$ IAM, $n=7$ e IAM + fasudil, $n=7$ ) Cuantificaciones están expresadas en relación con el grupo Sham (en número de veces). Las determinaciones están normalizadas con los niveles de GAPDH. Datos expresados como promedio \pm ES. Símbolos: $\# \#=p<0.05$ vs Sham (Newman Keuls post ANOVA significativo).
Figura 4

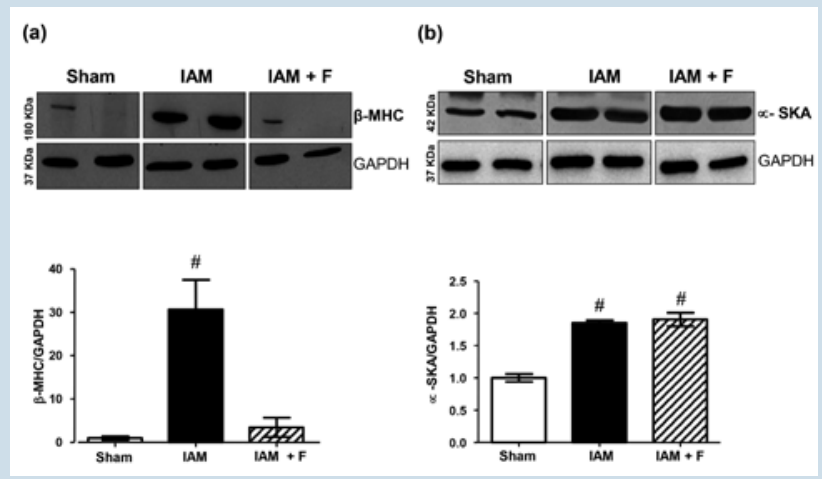

Efecto de la inhibición de ROCK sobre marcadores tempranos de hipertrofia cardíaca post IAM ( $\beta$-MHC y a-SKA). a) Imágenes representativas de WB de $\beta$-MHC en VI en ratas 1 semana post IAM en los 3 grupos experimentales (panel superior). Panel inferior: análisis densitométrico comparativo en los 3 grupos experimentales (Sham, $n$ $=5 ;$ IAM, $n=7$ e IAM + fasudil, $n=4$ ). b) Imágenes representativas de WB de a-SKA en VI en ratas 1 semana post IAM -MI en los 3 grupos experimentales (Panel superior). Panel inferior: análisis densitométrico comparativo en los 3 grupos experimentales ( $n=3 / g r u p o)$. Cuantificaciones expresadas en relación con el grupo Sham (en número de veces). Las determinaciones están normalizadas con los niveles de GAPDH. Datos expresados como promedio \pm ES. Símbolos: $\#=p<0.01$ vs Sham (Newman Keuls post ANOVA significativo).

en miocardio aumentaron 30 veces en comparación con el grupo control (y disminuyeron significativamente con fasudil ( $\mathrm{p}<0.001$ ). De manera similar, en el grupo IAM, los niveles de $\alpha$-SKA aumentaron en un $85 \%$ ( $p<0.001$ ), sin cambios después de la administración de fasudil.

El área de sección transversal de los cardiomiocitos en el grupo sham fue $167 \pm 3 \mu \mathrm{m} 2$ y fue significativamente mayor en un $10 \%$ en el grupo IAM ( $\mathrm{p}<0.05$ ). Fasudil administrado durante 7 días a ratas con IAM no modificó este parámetro en comparación con el grupo control.

Fibrosis miocárdica (Figura 5). Tanto en las áreas cercanas al IAM como en las áreas alejadas del IAM se observó un aumento significativo en la fracción volumétrica de colágeno (FVC) en el grupo IAM en comparación con el grupo control operado $(1,16 \pm 0,34$ versus $5,1 \pm 0,6 \%$ y $1.16 \pm 0.34$ versus $4.2 \pm 0.3 \%, \mathrm{n}=6, \mathrm{p}<0.001$, respectivamente). La fibrosis miocárdica se atenuó en el grupo IAM tratado con fasudil en ambas áreas del ventrículo izquierdo en comparación con el grupo IAM sin tratamiento.

\section{Fosforilación de ERK 42, ERK 44 y de GATA-4 en mio-} cardio. Debido al papel desempeñado por la vía de señalización ROCK /ERK /GATA-4 en el remodelado y en la 
Figura 5.

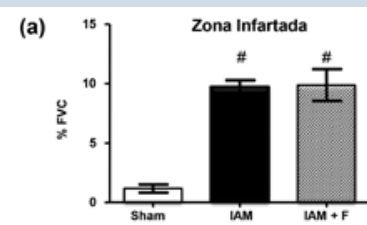

(b)

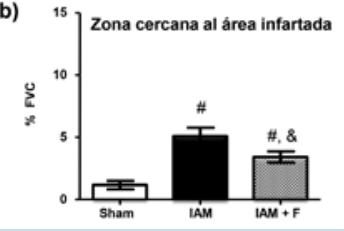

(c)

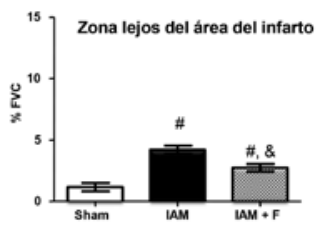

Efecto de la inhibición de ROCK sobre la fibrosis miocárdica una semana post IAM (morfometría). La fracción volumétrica de colágeno (FVC) determinada en secciones transversales de VI teñidas con Rojo Picrosirio la zona infartada (a), en la zona próxima al área infartada (b) y en la zona alejada del infarto (c) en los 3 grupos experimentales (Sham, $n=5 ;$ IAM, $n=6$ e IAM + fasudil, $n=5$ ). Datos expresados como promedio \pm ES. Símbolos: $\#=p<0.01$ vs Sham; $\&=p<0.01$ vs IAM (Newman Keuls post ANOVA significativo).

Figura 6.
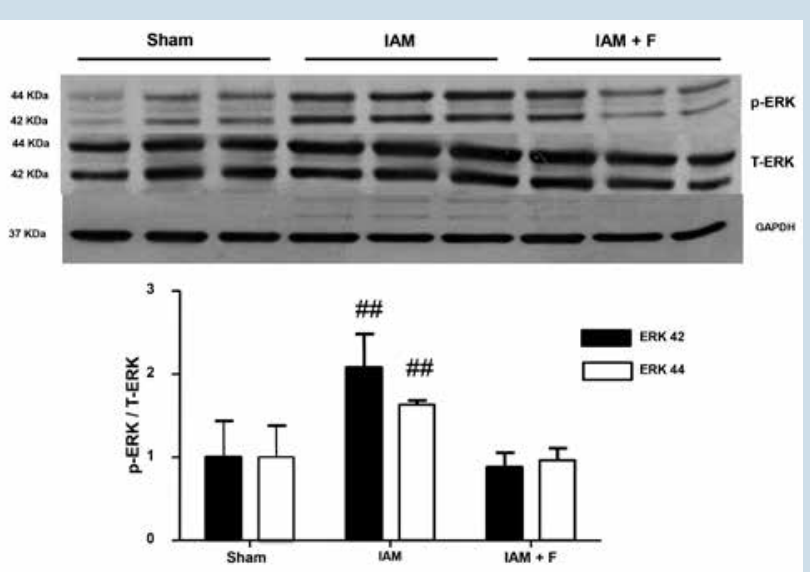

La inhibición de ROCK reduce la sobrexpresión cardíaca de ERK 42 y ERK 44 post IAM. Imágenes representativas de WB de ERK 42 y ERK 44 fosforilada y total en VI en ratas 1 semana post IAM en los 3 grupos experimentales (panel superior). Panel inferior: análisis densitométrico comparativo en los 3 grupos experimentales de ERK 42 (negro) y ERK 44 (blanco) fosforilada/total ( $n=3 /$ grupo). Cuantificaciones expresadas en relación con el grupo Sham (en número de veces). Las determinaciones están normalizadas con los niveles de GAPDH. Datos expresados como promedio \pm ES. Símbolos: \#\# $=p<0.05$ vs Sham (Newman Keuls post ANOVA significativo). contractilidad del miocardio, se determinaron estos niveles. El IAM aumentó significativamente los niveles de fosforilación de ERK 42 y ERK 44 en 2 y $63 \%$ respectivamente (Figura 6). En el grupo con IAM tratado con el inhibidor de ROCK estos niveles fueron similares a los observados en el grupo control. Además, el IAM produjo un aumento significativo de los niveles del factor de transcripción GATA-4 fosforilado en 2,9 veces (Figura 7), mientras que con fasudil los niveles fueron similares a los del grupo control. El IAM también indujo un aumento significativo en los niveles de proteína total de GATA-4 en 2,5 veces, sin cambios en el grupo IAM tratado con fasudil.

\section{Discusión:}

Las principales observaciones de este estudio en un modelo preclínico de IAM fueron: la disfunción sistólica VI está fuertemente asociada a la activación de ROCK cardíaca y a la fosforilación secundaria de las proteínas río abajo de ROCK que promueven remodelado ventricular como son $\beta$-MHC y la vía ERK/GATA-4. La inhibición de ROCK con fasudil mejoró significativamente la función sistólica, disminuyó la fibrosis miocárdica y

\section{Figura 7.}

(a)

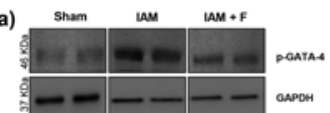

(b)
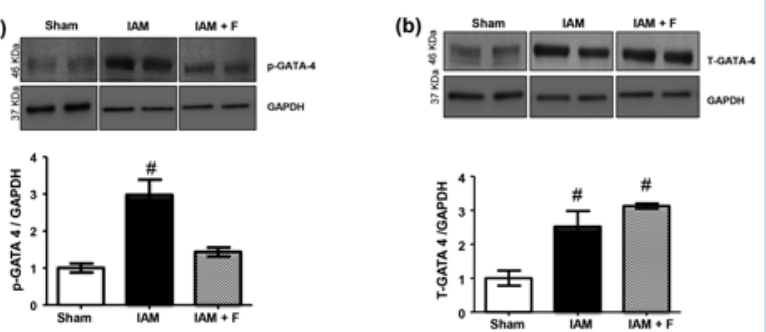

(c)

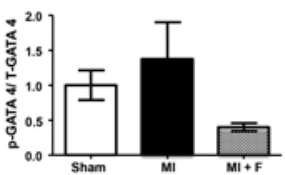

Fosforilación cardíaca de GATA 4 post IAM y efecto de fasudil. a) Imágenes representativas de WB de GATA 4 fosforilada en VI en ratas 1 semana post IAM en los 3 grupos experimentales (panel superior). Panel inferior: análisis densitométrico comparativo en los 3 grupos experimentales (Sham, $n=4$; IAM, $n=4$ e IAM + fasudil, $n=3$ ). b) Imágenes representativas de WB de GATA 4 total en VI en ratas 1 semana post IAM en los 3 grupos experimentales (panel superior). Panel inferior: análisis densitométrico comparativo en los 3 grupos experimentales c) análisis densitométrico de la relación p-GATA 4/T-GATA 4 en los 3 grupos. Las cuantificaciones se expresan en relación al grupo Sham (en número de veces). Las determinaciones están normalizadas con los niveles de GAPDH. Datos expresados como promedio \pm ES. Símbolos: $\#=p<0.01$ vs Sham (Newman Keuls post ANOVA significativo). 
normalizó los niveles de $\beta$-MHC y de fosforilación de ERK/GATA-4.

\section{Activación de ROCK y disfunción sistólica post IAM.} El método más reconocido para determinar la activación de ROCK es midiendo el nivel de fosforilación de una de sus proteínas blanco, MYPT1. MYPT1 es parte del complejo holoenzimático fosfatasa de la miosina, la subunidad reguladora de la unión de la miosina. La actividad de esta fosfatasa está regulada por el estado de fosforilación de los residuos de treonina 696 y 853 en MYPT1, que pueden ser fosforilados por ROCK ${ }^{28-30}$. Los estudios in vitro han establecido que la fosforilación en el residuo 853 es completamente evitada por los inhibidores de ROCK, mientras que el residuo T696 también puede ser fosforilado por otras quinasas

En el presente estudio, en ratas con disfunción sistólica temprana una semana post IAM, los niveles de fosforilación cardíaca de MYPT1 aumentaron significativamente y el inhibidor de ROCK fasudil redujo esta fosforilación, lo que indica el papel de la activación de ROCK cardíaca post IAM (y posiblemente en otros tipos de disfunción sistólica). La dosis y el método de administración de fasudil utilizados se han descrito previamente para evaluar inhibición de ROCK cardiovascular en roedores $13,14,31,32$.

Con el fin de caracterizar mejor la especificidad de la fosforilación de ROCK en este modelo, se determinó el nivel de fosforilación de ERM cardíaco, otro blanco directo de $\mathrm{ROCK}^{12,33}$, en el miocardio de VI no infartado en una muestra de los 3 grupos experimentales como se ha reportado previamente en ratones ${ }^{34}$. Se observó un aumento significativo en los niveles de fosforilación de ERM cardíaco una semana post IAM, lo que también se redujo con fasudil. Nuestros hallazgos en ERM son consistentes con observaciones sobre activación cardíaca de ROCK en ratones 4 días 34 y 4 semanas post IAM ${ }^{14}$ así como en el modelo de isquemia-reperfusión en la rata ${ }^{35}$. Las proteínas de ERM están estrechamente relacionadas con la dinámica del citoesqueleto y son componentes importantes de la estructura celular ${ }^{36,37}$, pero el rol de la fosforilación de ERM en células cardíacas no es algo bien conocido. En cardiomiocitos, la activación de ERM media los efectos de la activación del intercambiador de $\mathrm{Na}+/ \mathrm{H}+$ inducido por ácido ${ }^{37}$.

Además, observamos un aumento significativo en los niveles de las isoformas cardíacas ROCK1 y ROCK2, lo que no se había reportado previamente en este modelo experimental. El aumento de estas 2 isoformas de ROCK se ha observado experimentalmente en células de músculo liso vascular en hipertensión experimental y también en arterias pulmonares en situación de hipoxia y también como consecuencia de daño pulmonar. ${ }^{38}$

En ratas con diabetes inducida por estreptozotocina se ha observado un aumento de ambas isoformas cardíacas de $\mathrm{ROCK}^{39}$, y también en el modelo de isquemia-reperfusión ${ }^{40}$. Previamente, en este modelo de IAM, localizamos niveles aumentados de ROCK2 (y el efecto de la inhibición de ROCK) dentro de los cardiomiocitos usando inmunofluorescencia, en paralelo con aumento de $\beta$-MHC, lo que apoya el papel de ROCK2 en la hipertrofia cardíaca ${ }^{27}$.

ROCK1 participa en procesos celulares que caracterizan el remodelado cardíaco patológico como fibrosis, hipertrofia y apoptosis de cardiomiocitos, que conducen a dilatación y disfunción ventricular y a insuficiencia cardíaca. ROCK1 parece mediar la señalización alterada de supervivencia dependiente de ERK y de Akt, el aumento de citoquinas fibrogénicas y la expresión alterada de AC5/6 inducidas por estímulos hipertróficos ${ }^{12}$. Por otro lado, estudios genéticos que utilizan ratones knockout para ROCK2 específica en corazón han demostrado que ROCK2 está involucrado en hipertrofia de cardiomiocitos, en apoptosis y en fibrosis cardíaca durante la hipertrofia cardíaca compensatoria 41,11 .

Estudios genéticos recientes indican que las isoformas de ROCK pueden tener funciones distintas en el remodelado cardíaco. A este respecto, queda por determinar si los efectos beneficiosos de los inhibidores de ROCK están mediados por la inhibición de ROCK1, ROCK2 o de ambos. En este sentido, estudios con ratones knockout condicional de ROCK1 y ROCK2, así como el uso de inhibidores específicos de cada isoforma podrían proporcionar nuevos conocimientos sobre su rol en el IAM y en el remodelado cardíaco.

Remodelado ventricular. $\mathrm{La}$ injuria miocárdica se asocia a reexpresión de genes normalmente expresados en la vida fetal temprana como $\beta$-MHC y $\alpha$-SKA, que son marcadores tempranos de hipertrofia ${ }^{2,42,43}$. En nuestras ratas con IAM, observamos un aumento significativo de los niveles cardíacos de $\beta$-MHC y $\alpha$-SKA, consistente con reportes anteriores ${ }^{2}, 42,43$. Fasudil redujo los niveles de $\beta$-MHC a niveles normales, lo que indica que la activación de ROCK contribuye al remodelado adicionalmente a través de la expresión de beta-MHC. Fasudil no redujo los niveles cardíacos de $\alpha$-SKA, lo que sugiere que la expresión de esta proteína post IAM es indepen- 
diente de la activación de ROCK.

Se ha propuesto que en la hipertrofia cardíaca por sobrecarga de presión pueden existir dos poblaciones distintas de cardiomiocitos dentro del corazón hipertrófico: una que es hipertrófica y que expresa predominantemente $\alpha$-MHC y una segunda población no hipertrófica que expresa $\alpha$ y $\beta$-MHC 44,45 . Desde esta perspectiva, la reexpresión de $\beta$-MHC sería un marcador celular de 'normotrofia ' bajo condiciones que inducen hipertrofia cardíaca, lo que haría a estos cardiomiocitos resistentes a un aumento de su tamaño de manera que esta reexpresión de $\beta$-MHC sería de hecho protectora ${ }^{44,45}$. La reexpresión de $\beta$-MHC se produce en grupos discretos de miocitos dentro del subendocardio lo que parece sugerir que la inducción de $\beta$-MHC no es una consecuencia obligatoria de hipertrofia celular; también las células que expresan $\beta$-MHC en el corazón hipertrófico se distribuyen predominantemente en agrupaciones dentro y también rodeando focos de fibrosis 45 .

Para evaluar con más detalle el mecanismo por el cual la activación de ROCK regula la expresión de $\beta$-MHC cardíaca, nos hemos centrado en el factor de transcripción GATA-4, que se transloca al núcleo y se une a secuencias de consenso $[(\mathrm{A} / \mathrm{T}) \mathrm{GATA}(\mathrm{A} / \mathrm{G})]$ en los promotores de genes que codifican proteínas como $\beta$-MHC, el receptor de angiotensina II tipo 1 , endotelina- ${ }^{39}$ y también troponina $^{46}$. La activación transcripcional de GATA-4 depende de la fosforilación en serina 105 y está mediada principalmente por la proteína quinasa activada por mitógeno (MEK 1) y ERK1/2 47,16,42,43. ROCK es un regulador directo de la vía ERK1/2, que fosforila y activa a GATA-4 a nivel cardíaco ${ }^{42}$. En este estudio hemos observado un aumento en los niveles de fosforilación de ERK1/2 en ratas con IAM y también en los niveles de fosforilación en el residuo de serina 105 en GATA-4 miocárdica. Ambos cambios se redujeron con fasudil, lo que indica el rol de la activación de ROCK en estos procesos. La activación de la vía ROCK/ERK/GATA-4 cardíaca en este contex to también es consistente con el aumento en la expresión de $\beta$-MHC en el grupo con IAM.

Junto con la hipertrofia cardíaca, en este modelo de IAM (grande), aumentó el colágeno intersticial en la zona no infartada del ventrículo izquierdo. Fasudil revertió la fibrosis miocárdica, consistente con el rol de la activación de ROCK en la fibrosis miocárdica ${ }^{14-18,34}$. En ratones con deleción del gen ROCK1, se ha identificado el rol de esta isoforma en el desarrollo de fibrosis cardíaca en respuesta a la isquemia reperfusión ${ }^{9}$ y también en ratones haploinsuficientes ROCK1 (+/-) estimulados con angiotensina $\mathrm{II}^{18}$.

Estos resultados in vivo (aumento de la fosforilación cardíaca de las proteínas blanco de ROCK y su reducción con fasudil, junto con mejoría de la función sistólica) demuestran que los efectos observados son bastante específicos de esta quinasa. Por otro lado, el aumento en los niveles de cardíacos de MYPT1 y $\beta$-MHC y su disminución con fasudil, sugiere que ROCK es un regulador importante en las modificaciones postranduccionales de estas proteínas asociadas a remodelado y a contractilidad miocárdica $^{48-50}$.

Limitaciones. Habiendo utilizado un modelo in vivo y observado cambios cardíacos inducidos en un contexto fisiopatológico integrado, el papel de otras vías de señalización no se evaluó. Además, las modificaciones observadas, 1 semana post IAM, podrían no estar presentes en el largo plazo. Por otro lado, en ratones con IAM de 4 semanas también se observan los efectos deletéreos de la activación de ROCK en el remodelado y función sistólica $\mathrm{VI}^{18}$, de manera que las alteraciones observadas posiblemente son más que transitorias. También, hemos utilizado todo el VI en lugar de la zona no infartada del VI para las determinaciones moleculares y bioquímicas. Sin embargo, para los efectos de interpretar los resultados, las mismas mediciones se realizaron en ambos grupos experimentales, con áreas de infarto similares, presentando el grupo IAM mayor remodelado en la zona no infartada en comparación con el grupo tratado con fasudil. Por lo tanto, es razonable proponer que los cambios moleculares observados muy probablemente tuvieron lugar en la zona no infartada. Tampoco realizamos estudios de expresión génica, pero se analizó cuantitativamente el rol de varias proteínas fosforiladas que son importantes en el remodelado y en la función ventricular izquierda, y que finalmente dan cuenta de las modificaciones de un fenotipo.

En conclusión, mecanismos relevantes por los cuales la activación temprana de ROCK post IAM deteriora la función sistólica VI son la estimulación de hipertrofia y fibrosis, la fosforilación de ERK42, $\mathrm{ERK}^{44}$ y del factor de transcripción GATA-4 a nivel miocárdico. La inhibición de ROCK post IAM mejora significativamente la función sistólica VI y normaliza estos mecanismos alterados. 
Referencias

1. PARAJULI, N., YUAN, Y., ZHENG, X., BEDJA, D, CAI, Z.P. Phosphatase PTEN is critically involved in post-myocardial infarction remodeling through the Akt/ interleukin-10 signaling pathway. Basic Res Cardiol 2012;107:248.

2. SUTTON, M.G., SHARPE, N. Left ventricular remodeling after myocardial infarction: pathophysiology and therapy. Circulation 101; 2000:2981-2988.

3. HAUDEK, S.B., GUPTA, D., DEWALD, O., SCHWARTZ R.J., WEI, L., TRIAL, J. et al. Rho kinase-1 mediates cardiac fibrosis by regulating fibroblast precursor cell differentiation. Cardiovasc Res 2009;83:511-518.

4. WALKER, L.A., WALKER, J.S., AMBLER, S.K., BUTTRICK, P.M. Stage-specific changes in myofilament protein phosphorylation following myocardial infarction in mice. J Mol Cell Cardiol 2010; 48:1180-1186.

5. JALIL JE, OCARANZA MP. Regression of cardiovascular remodeling in hypertension: Novel relevant mechanisms World J Hypertens 2016; 6: 1-17

6. SHIMOKAWA $\mathrm{H}$, SUNAMURA $\mathrm{S}$, SATOH $\mathrm{K}$. RHOA/RHO-KINASE in the Cardiovascular System. Circ Res. 2016;118:352-66.

7. JALIL, J., LAVANDERO, S., CHIONG, M., OCARANZA, M.P. RHO/RHO kinase signal transduction pathway in cardiovascular disease and cardiovascular remodeling. Rev Esp Cardiol 2005;58:951-961.

8. SURMA, M., WEI, L., SHI, J. Rho kinase as a therapeutic target in cardiovascular disease. Future Cardiol 2011; 7:657-671.

9. SATOH, K., FUKUMOTO, Y., SHIMOKAWA, H. RHO-KINASE: important new therapeutic target in cardiovascular diseases. Am J Physiol Heart Circ Physiol 2011;301:,H287-H296.
10. LOIRAND, G., SAUZEAU, V., Pacaud, P. Small G proteins in the cardiovascular system: physiological and pathological aspects. Physiol Rev 2013; 93:1659-720

11. SHI, J., WEI, L. Rho kinases in cardiovascular physiology and pathophysiology: the effect of fasudil. J Cardiovasc Pharmacol 2013; 62:341-354.

12. SHI, J., ZHANG, L., WEI, L. Rho-kinase in development and heart failure: insights from genetic models. Pediatr Cardiol 2011;32:297-304.

13. RIVERA, P., OCARANZA, M.P., LAVANDERO, S, JALIL, J.E. Rho kinase activation and gene expression related to vascular remodeling in normotensive rats with high angiotensin I converting enzyme levels. Hypertension 2007;50:792-798.

14. HATTORI, T., SHIMOKAWA, H., HIGASHI, M., HIROKI, J., MUKAI, Y., TSUTSUI, H. et al. (2004) Long-term inhibition of Rho-kinase suppresses left ventricular remodeling after myocardial infarction in mice. Circulation 109:2234-2239.

15. LI, Q., XU, Y., LI, X., GUO, Y., LIU, G. Inhibition of Rho-kinase ameliorates myocardial remodeling and fibrosis in pressure overload and myocardial infarction: role of TGF- $\beta 1$-TAK1. Toxicol Lett 2012; 211:91-97.

16. WANG, N., GUAN, P., ZHANG, J.P., LI, Y.Q., CHANG, Y.Z., SHI, Z.H. et al. Fasudil hydrochloride hydrate, a Rho-kinase inhibitor, suppresses isoproterenol-induced heart failure in rats via JNK and ERK1/2 pathways. J Cell Biochem 2011;112:1920-1929.

17. VARGAS, M., RIVERA, P., OCARANZA, M.P., JALIL, J. La vía de señalización Rho A/ Rho Kinasa se encuentra activada en el miocardio en ratas con fibrosis cardíaca inducida por isoprotenerol. Rev Chil Cardiol 2009; 28: 81-89.

18. RIKITAKE, Y., OYAMA, N., WANG, C.Y., NOMA, 
K., SATOH, M., KIM, H.H. et al. Decreased perivascular fibrosis but not cardiac hypertrophy in ROCK1+/- haploinsufficient mice. Circulation 2005; 112:2959-2965.

19. SHI, J., ZHANG, Y.W., YANG, Y., ZHANG, L., WEI, L. ROCK1 plays an essential role in the transition from cardiac hypertrophy to failure in mice. J Mol Cell Cardiol 2010; 49:819-828.

20. JIANG, Z.H., ZHANG, T.T., ZHANG, J.F. Protective effects of fasudil hydrochloride post conditioning on acute myocardial ischemia/reperfusion injury in rats. Cardiol J 2013; 20:197-202.

21. DEMIRYÜREK, S., KARA, A.F., CELIK, A., BABÜL, A., Tarakçioglu, M., Demiryürek, A.T. Effects of fasudil, a Rho-kinase inhibitor, on myocardial preconditioning in anesthetized rats. Eur J Pharmacol 2005; 527:129-140.

22. ZHANG, J., BIAN, H.J., LI, X.X., LIU, X.B., SUN, J.P., LI, N.et al. ERK-MAPK signaling opposes rho-kinase to reduce cardiomyocyte apoptosis in heart ischemic reconditioning. Mol Med 2010; 16:307-315.

23. SHI, J., ZHANG, Y.W., SUMMERS, L.J., DORN, G.W.,Wei L. Disruption of ROCK1 gene attenuates cardiac dilation and improves contractile function in pathological cardiac hypertrophy. J Mol Cell Cardiol 2008; 44:551-560.

24. DEL RE, D.P., MIYAMOTO, S., BROWN, J.H. RHOA/Rho-kinase up-regulate Bax to activate a mitochondrial death pathway and induce cardiomyocyte apoptosis. J Biol Chem 2007; 282:8069-8078.

25. MIZUTANI, H., OKAMOTO, R., MORIKI, N., KONISHI, K., TANIGUCHI, M., FUJITA, S. et al. Overexpression of Myosin phosphatase reduces $\mathrm{Ca}(2+)$ sensitivity of contraction and impairs cardiac function. Circ J 2010; 74,120-128.

26. DE SIMONE, G., DEVEREUX, R.B., GANAU, A., HAHN, R.T., SABA, P.S., MUREDDU, G.F. et al. Estimation of left ventricular chamber and stroke volume by limited M-mode echocardiography and validation by two-dimensional and Doppler echocardiography. Am J Cardiol 1996;78:801-807.
27. MERA C, GODOY I, RAMÍREZ R, MOYA J, OCARANZA MP, JALIL JE. Mechanisms of favorable effects of Rho kinase inhibition on myocardial remodeling and systolic function after experimental myocardial infarction in the rat. Ther Adv Cardiovasc Dis. 2016;10(1):4-20.

28. ICHIKAWA, K., ITO, M., HARTSHORNE, D.J. Phosphorylation of the large subunit of myosin phosphatase and inhibition of phosphatase activity. J Biol Chem 1996; 271:4733-4740.

29. FENG, J., ITO, M., ICHIKAWA, K., ISAKA, N., NISHIKAWA, M., HARTSHORNE, D.J. et al. Inhibitory phosphorylation site for Rho-associated kinase on smooth muscle myosin phosphatase. J Biol Chem 1999; 274:37385-37390.

30. KHROMOV, A., CHOUDHURY, N., STEVENSON, A.S., SOMLYO, A.V., ETO, M. Phosphorylation dependent autoinhibition of myosin light chain phosphatase accounts for $\mathrm{Ca} 2+$ sensitization force of smooth muscle contraction. J Biol Chem 2009; 284:21569-21579.

31. KAGIYAMA, S., MATSUMURA, K., GOTO, K., OTSUBO, T., IIDA, M. Role of Rho kinase and oxidative stress in cardiac fibrosis induced by aldosterone and salt in angiotensin type 1a receptor knockout mice. Regul Pept 2010; 160:133-139.

32. OCARANZA, M.P., RIVERA, P., NOVOA, U., PINTO, M., GONZÁLEZ, L., CHIONG, M. et al. Rho kinase inhibition activates the homologous angiotensin-converting enzyme-angiotensin-(1-9) axis in experimental hypertension. J Hypertens 2011; 29:706-715.

33. HÉBERT, M., POTIN, S., SEBBAGH, M., BERTOGLIO, J., BRÉARD, J., HAMELIN, J. Rho-ROCK-dependent ezrin-radixin-moesin phosphorylation regulates Fas-mediated apoptosis in Jurkat cells. J Immunol 2008; 181:5963-5973.

34. CHAU, V.Q., SALLOUM, F.N., HOKE, N.N., ABBATE, A., KUKREJA, R.C. Mitigation of the progression of heart failure with sildenafil involves inhibition of RhoA/Rho-kinase pathway. Am J Physiol Heart Circ Physiol 2011;300:H2272-H2279. 
35. LI, Y., ZHU, W., TAO, J., XIN, P., LIU, M., LI, J. et al. Fasudil protects the heart against ischemia-reperfusion injury by attenuating endoplasmic reticulum stress and modulating SERCA activity: the differential role for PI3K/Akt and JAK2/STAT3 signaling pathways. PLoS One 2012; 7:e48115.

36. NIGGLI, V., ROSSY, J.R. Ezrin/radixin/moesin: versatile controllers of signaling molecules and of the cortical skeleton. Int J Biochem Cell Biol 2008;40:344-349.

37. DARMELLAH, A., RÜCKER-MARTIN, C., FEUVRAY, D. ERM proteins mediate the effects of $\mathrm{Na}+\mathrm{H}+$ exchanger (NHE1) activation in cardiac myocytes. Cardiovasc Res 2009;81:294-300

38. MCNAMARA, P.J., MURTHY, P., KANTORES, C., TEIXEIRA, L., ENGELBERTS, D., VAN VLIET, T. et al. Acute vasodilator effects of Rho-kinase inhibitors in neonatal rats with pulmonary hypertension unresponsive to nitric oxide. Am J Physiol Lung Cell Mol Physiol 2008;294:L205- L213.

39. GUO, R., LIU, B., ZHOU, S., ZHANG, B., XU, Y. The protective effect of fasudil on the structure and function of cardiac mitochondria from rats with type 2 diabetes induced by streptozotocin with a high-fat diet is mediated by the attenuation of oxidative stress. Biomed Res Int 2013:430791.

40. GUO, J., WANG, S.B., YUAN, T.Y., WU, Y.J., YAN, Y., LI, L. et al. Coptisine protects rat heart against myocardial ischemia/reperfusioninjury by suppressing myocardial apoptosis and inflammation. Atherosclerosis 2013;231:384-391.

41. OKAMOTO, R., LI, Y., NOMA, K., HIROI, Y., LIU, P.Y., TANIGUCHI, M. et al. FHL2 prevents cardiac hypertrophy in mice with cardiac-specific deletion of ROCK2. FASEB J 2013;27:1439- 1449.

42. YANAZUME, T., HASEGAWA, K., WADA, H., MORIMOTO, T., ABE, M., KAWAMURA, T. et al Rho/
ROCK pathway contributes to the activation of extracellular signal-regulated kinase/GATA-4 during myocardial cell hypertrophy. J Biol Chem 2002; 277:8618-8625.

43. AKAZAWA, H., KOMURO, I. Roles of cardiac transcription factors in cardiac hypertrophy. Circ Res 2003;92:1079-1088.

44. LOPEZ, J., MYAGMAR, B., SWIGART, P., MONTGOMERY,M., HAYNAM, S., BIGOS, M. et al. Beta Myosin heavy chain is induced by pressure overload in a minor subpopulation of smaller mouse cardiac myocytes. Circ Res 2011;109: 629-638.

45. PANDYA, K., KIM, H. and Smithies, O. Fibrosis, not cell size, delineates beta myosin heavy chain reexpression during cardiac hypertrophy and normal aging in vivo. Proc Natl Acad Sci USA 2006;103:16864-16869.

46. MURPHY, A.M., THOMPSON, W.R., PENG, L.F., JONES, L. Regulation of the rat cardiac troponin I gene by the transcription factor GATA-4. Biochem J 1997;322:393-401.

47. CAI, J., YI, F.F., BIAN, Z.Y., SHEN, D.F., YANG, L., YAN, L. Crocetin protects against cardiac hypertrophy by blocking MEK-ERK1/2 signaling pathway. J Cell Mol Med 2009;13: 909-925.

48. BERENJENO, I.M., BUSTELO, X.R. Identification of the Rock-dependent transcriptome in rodent fibroblasts. Clin Transl Oncol. 2008;10:726-738.

49. PENG, J., ZHANG, G., WANG, Q., HUANG, J., MA, H., ZHONG, Y. ROCK cooperated with ET-1 to induce epithelial to mesenchymal transition through SLUG in human ovarian cancer cells. Biosci Biotechnol Biochem. 2012; 76:42-47.

50. VLASBLOM, R., MULLER, A., BECKERS, C.M., van Nieuw Amerongen, G.P., Zuidwijk, M.J., van Hardeveld, C. RhoA-ROCK signaling is involved in contraction-mediated inhibition of SERCA2a expression in cardiomyocytes. Pflugers Arch. 2009;458:785-793. 